\title{
Racial disparities in papillary thyroid microcarcinoma survival
}

\author{
U C MEGWALU $^{1}$, A T SAINI ${ }^{2}$ \\ ${ }^{1}$ Department of Otolaryngology - Head and Neck Surgery, Stanford University School of Medicine, California, \\ and ${ }^{2}$ Department of Otolaryngology - Head and Neck Surgery, Icahn School of Medicine at Mount Sinai, \\ New York, USA
}

\begin{abstract}
Objective: To evaluate the impact of race on survival in patients with papillary thyroid microcarcinoma.

Methods: The study cohort included 17668 patients diagnosed with papillary thyroid microcarcinoma between 1988 and 2009, identified in the Surveillance, Epidemiology, and End Results 18 database of the National Cancer Institute.

Results: Black patients had lower overall survival than other racial groups $(p<0.001)$. Black patients had significantly worse overall survival (hazard ratio $=2.59$ ) after adjusting for sex, marital status, age, year of diagnosis, multifocal disease and type of surgery. A subset analysis of Black patients revealed no significant difference in overall survival for total thyroidectomy versus lobectomy $(p=0.15)$.

Conclusion: Black race is a negative prognostic factor in thyroid cancer, which cannot be explained by advanced disease stage. Further research on mechanisms by which race affects survival is needed to reveal areas of opportunity for interventions aimed at reducing health disparities in cancer care.
\end{abstract}

Key words: Thyroid Neoplasms; Health Status Disparities; Minority Health; SEER Program

\section{Introduction}

The incidence of thyroid cancer is rising, mostly because of the rapid increase in the incidence of small papillary thyroid cancers. ${ }^{1-4}$ The prognosis of localised thyroid cancer is excellent. However, age, sex, tumour size, extrathyroidal extension, histological grade and type, local invasion, multicentricity, presence of metastatic disease, and completeness of surgical resection are known to be independent predictors of survival in thyroid malignancies. ${ }^{5-7}$

Racial disparities are becoming increasingly apparent in a variety of head and neck cancers. ${ }^{8-11}$ Few studies have looked specifically at racial disparities in thyroid malignancies. A recent study evaluating the impact of race and ethnicity on thyroid cancer presentation found that African Americans had larger tumours compared with other racial groups. Another study found that racial minority groups and patients with low socioeconomic status had a higher likelihood of presenting with metastatic disease, and had an increased risk of mortality. ${ }^{12}$ Yu et al. evaluated risk factors for mortality in patients with papillary thyroid microcarcinoma. ${ }^{13}$ They found that African American or minority race was associated with an increased risk of mortality. However, their cohort included patients with nodal and distant metastasis, which are known poor prognostic factors. Our study aimed to evaluate the impact of race on survival in patients with localised papillary thyroid microcarcinoma using a large population-based cancer database.

\section{Materials and methods}

Data were extracted from the Surveillance, Epidemiology, and End Results ('SEER') 18 database of the National Cancer Institute, which includes data obtained from 18 population-based registries. Seven registries (Connecticut, Detroit, Hawaii, Iowa, New Mexico, San Francisco (Oakland) and Utah) joined the Surveillance, Epidemiology, and End Results Program in 1973; two registries (Seattle (Puget Sound) and Atlanta) joined in 1974 and 1975, respectively; four registries (Los Angeles, San Jose (Monterey), Rural Georgia and the Alaska Native Tumor Registry) joined in 1992; and five registries (Greater California, Kentucky, Louisiana, New Jersey and Greater Georgia) joined in 2000. ${ }^{14}$

The study cohort included patients diagnosed with early-stage papillary thyroid carcinoma between 1988 and 2009 with a tumour size of $1 \mathrm{~cm}$ or less. The International Classification of Diseases for Oncology 
code used was C73.9 for thyroid gland. Exclusion criteria included regional or distant metastasis, and multiple primaries. Patients who did not undergo thyroidectomy were also excluded. Race was recorded in the Surveillance, Epidemiology, and End Results database as 'White', 'Black', 'Other: American Indian, AK Native, Asian/Pacific Islander' or 'Unknown'. Marital status was grouped as 'married' (including common law) or 'single' (never married, divorced, widowed). Therapy was coded as thyroid lobectomy or total thyroidectomy.

The Surveillance, Epidemiology, and End Results computer software (SEER*Stat 8.1.5) was used to extract data from the Surveillance, Epidemiology, and End Results database. IBM SPSS ${ }^{\circledR}$ version 20 software was used for statistical analysis. Survival analysis was performed using Kaplan-Meier analysis. The primary outcome measure was overall cumulative survival. The primary independent variable was race. A Cox proportional hazards regression model was used for multivariable survival analysis. Race, sex, marital status, age, year of diagnosis, mode of therapy (total thyroidectomy $v s$ lobectomy) and multifocal disease were entered a priori into the model. A $p$ value of less than 0.05 was considered statistically significant. This study was exempt from the Icahn School of Medicine at Mount Sinai Institutional Review Board review because it was conducted using de-identified public data.

\section{Results}

From 1988 to 2009, the Surveillance, Epidemiology, and End Results database identified a total of 17668 patients who met the inclusion criteria. The patient characteristics are displayed in Table I. No patient had extrathyroidal extension. Information on marital status was missing for 678 patients and these patients were excluded from the multivariable analysis. Results of the univariate analysis are shown in Table II. Overall survival differed by race $(p<$ $0.001)$. Black patients had lower overall survival than

\begin{tabular}{ll}
\multicolumn{2}{c}{ TABLE I } \\
PATIENT CHARACTERISTICS \\
\hline Characteristic & \multicolumn{1}{c}{ Value } \\
\hline Sex $(n(\%))$ & \\
- Female & \\
- Male & $15008(84.9)$ \\
Mean age (years) & $2660(15.1)$ \\
Race $(n(\%))$ & 48.7 \\
- White & $14964(84.7)$ \\
- Black & $1096(6.2)$ \\
- Other & $1608(9.1)$ \\
Marital status $(n(\%))$ & $11896(67.3)$ \\
- Married & $5095(28.8)$ \\
- Single & \\
Therapy $(n(\%))$ & $4848(27.4)$ \\
- Lobectomy & $12820(72.6)$ \\
- Total thyroidectomy & $1618(9.2)$ \\
\hline Multifocal disease $(n(\%))$ & \\
\hline
\end{tabular}

\begin{tabular}{lccc}
\multicolumn{3}{c}{ TABLE II } \\
\multicolumn{3}{c}{ IMPACT OF RACE ON OVERALL SURVIVAL } \\
\cline { 2 - 3 } Race & \multicolumn{2}{c}{ Overall survival (\%) } & $p$ \\
\cline { 2 - 3 } & 5-year & 10-year & \\
\hline White & 98.0 & 95.4 & $<0.001$ \\
Black & 94.6 & 89.4 & \\
Other & 98.8 & 95.7 & \\
\hline
\end{tabular}

White patients and patients classified as 'Other' (Figure 1).

The results of the multivariable analysis are shown in Table III. Black patients had significantly worse overall survival (hazard ratio $=2.59$ ) after adjusting for sex, marital status, age, year of diagnosis, multifocal disease and type of surgery. Female sex (hazard ratio $=0.72$ ) and married status (hazard ratio $=0.53$ ) were associated with improved overall survival, while age (hazard ratio $=1.09$ ) was associated with worse overall survival. Overall survival improved over time (hazard ratio $=0.94$ for each subsequent year of diagnosis).

A subset analysis was performed comparing total thyroidectomy versus lobectomy in Black patients. There was no significant difference in overall survival between patients treated with total thyroidectomy and those treated with lobectomy (five-year overall survival rates were 95.9 per cent and 91.4 per cent respectively, $p=0.15$ ). On multivariable analysis, there was no difference in overall survival between the two groups after adjusting for sex, marital status, age, year of diagnosis and multifocal disease (hazard ratio $=1.11,95$ per cent confidence interval $=0.61-2.02, p=0.74$ ).

\section{Discussion}

The results of this study show that race has an impact on survival in patients with papillary thyroid

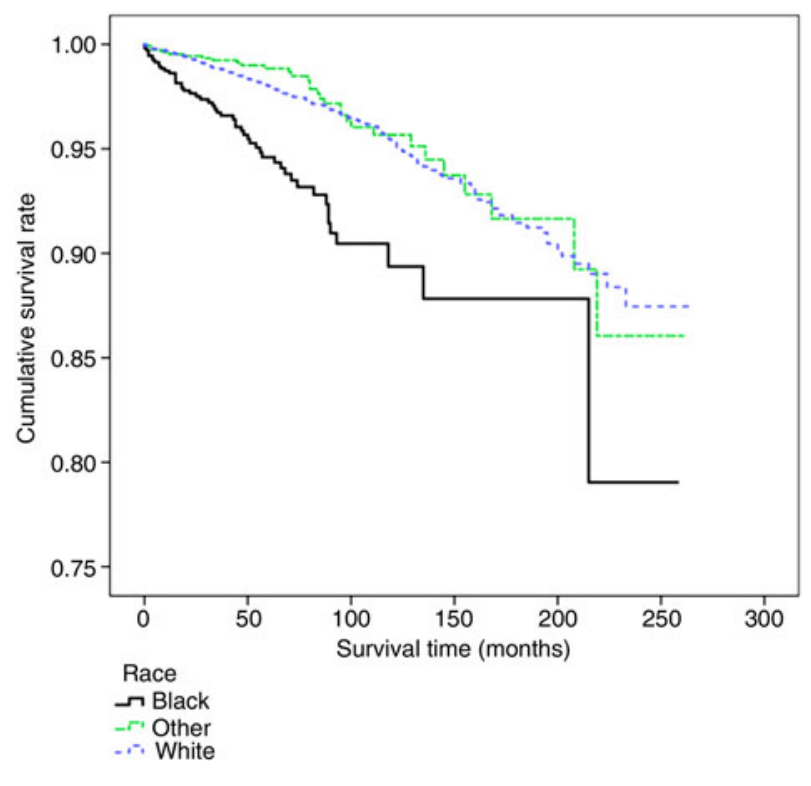

FIG. 1

Overall survival according to race. 


\begin{tabular}{|c|c|c|c|c|c|}
\hline \multicolumn{6}{|c|}{ TABLE III } \\
\hline Variable & Co-efficient (SE) & Wald chi-square & Hazard ratio & $95 \% \mathrm{CI}$ & $p$ \\
\hline Race: Black & $0.95(0.16)$ & 37.94 & 2.59 & $1.92-3.51$ & $<0.001$ \\
\hline Race: other & $-0.13(0.18)$ & 0.51 & 0.88 & $0.61-1.26$ & 0.47 \\
\hline Female & $-0.33(0.06)$ & 30.30 & 0.72 & $0.64-0.81$ & $<0.001$ \\
\hline Married & $-0.64(0.11)$ & 36.66 & 0.53 & $0.43-0.65$ & $<0.001$ \\
\hline Age & $0.08(0.004)$ & 461.12 & 1.09 & $1.08-1.10$ & $<0.001$ \\
\hline Year of diagnosis & $-0.07(0.01)$ & 25.78 & 0.94 & $0.91-0.96$ & $<0.001$ \\
\hline Multifocal disease & $0.05(0.11)$ & 0.26 & 1.06 & $0.86-1.30$ & 0.61 \\
\hline Lobectomy & $-0.11(0.14)$ & 0.58 & 0.90 & $0.69-1.18$ & 0.45 \\
\hline
\end{tabular}

$\mathrm{SE}=$ standard error; $\mathrm{CI}=$ confidence interval

microcarcinoma. Black patients had significantly worse survival, even after adjusting for sex, marital status, age, year of diagnosis, multifocal disease and mode of therapy. On multivariable analysis, female sex and married status were associated with improved survival. A subset analysis of Black patients revealed no difference in survival between those treated with total thyroidectomy and those treated with lobectomy.

Few studies have looked specifically at the impact of race on survival for patients with thyroid malignancies. Moo-Young et al. evaluated the impact of race and ethnicity on thyroid cancer presentation. ${ }^{15}$ They reviewed 18 047 cases of thyroid cancer identified within the Surveillance, Epidemiology, and End Results 17 database from 1996 to 2006. They found that African Americans younger than 45 years had the largest tumour size. However, the risk of lymph node metastasis was lowest in this group. They found no difference between ethnic groups in terms of capsular invasion and stage. Harari et al. investigated racial and socioeconomic disparities in well-differentiated thyroid cancer. ${ }^{12}$ Their study included 25945 patients from the California Cancer Registry diagnosed with thyroid cancer from 1999 to 2008. They found that racial minority groups and patients with low socioeconomic status had a higher likelihood of presenting with metastatic disease. Similar to our study, they also found that Black patients had the lowest survival rates. Yu et al. evaluated risk factors for mortality in patients with papillary thyroid microcarcinoma. ${ }^{13}$ The study included 18445 patients from the Surveillance, Epidemiology, and End Results database. Similar to our study, they found that age greater than 45 years, male sex, and African American or minority race were associated with increased risk of mortality. As expected, they also found that nodal metastasis and distant metastasis were associated with increased risk of mortality. In contrast, our study excluded patients with nodal or distant metastasis. Even then, an increased risk of mortality was noted in this cohort of patients, who are usually expected to have excellent prognosis.

The results of these studies are consistent with findings from other studies that show lower survival rates for African Americans with head and neck cancer compared with other races. A study by Chen and Halpern evaluated factors predictive of survival in advanced laryngeal cancer using data from the National Cancer Database. ${ }^{8}$ Similar to our study, they found that female sex and White race positively influenced survival. Shavers et al. examined racial and ethnic variation in tumour characteristics, treatment practices, and survival in head and neck cancer using the Surveillance, Epidemiology, and End Results database. ${ }^{9}$ They found that African Americans had worse survival compared with Hispanic or White patients. Furthermore, African Americans tended to be diagnosed with a more advanced stage of disease and had a lower likelihood of receiving treatment, which may have influenced the differences in survival.

It is unclear why Black race negatively influenced survival in our cohort. Many studies on racial disparities in head and neck cancer attribute some of the difference in survival to more advanced disease in Black patients. However, this does not explain the racial differences in survival in our study, given that all the patients in our cohort presented with microcarcinoma in the absence of regional or distant metastasis, and all patients received cancer-directed surgery. Furthermore, the survival gap remained even after adjusting for sex, marital status, age, year of diagnosis, multifocal disease and mode of therapy. The difference in survival may be because of racial differences in the quality of care received. A recent study investigated the effect of surgeon volume on racial disparities in thyroid surgery. ${ }^{16}$ The study utilised the nationwide in-patient sample, and included 106314 thyroid and parathyroid surgical procedures performed from 2003 to 2009 . The authors found that African Americans had higher complication and in-hospital mortality rates compared with other racial groups. They also found that higher surgeon volume was associated with improved outcomes. However, African Americans had less access to intermediate- and highvolume surgeons. Unfortunately, the impact of quality of care cannot be determined from our data.

Another reason for the difference in survival could be that papillary thyroid microcarcinoma is more aggressive in Black patients because of differences in tumour biology and genetic variation. However, there is currently no evidence for this in the literature. If this were the case, total thyroidectomy might prove beneficial in this group. We performed a subset analysis of Black 
patients, comparing total thyroidectomy against thyroid lobectomy. There was no difference in overall survival between the two treatment groups. In their study, Yu et al. identified African American or minority race as one of the risk factors for mortality in patients with papillary thyroid microcarcinoma. ${ }^{13}$ Similar to our study, they compared total thyroidectomy against thyroid lobectomy in patients with two or more risk factors. Contrary to our study, they found that total thyroidectomy provided a survival advantage in this cohort of patients.

Our study showed a survival advantage for married patients, which is consistent with findings from other studies on head and neck cancer. A recent study evaluating the impact of demographic and socioeconomic factors on survival in major salivary gland cancer found that married patients had higher survival rates than single patients. ${ }^{10}$ Similarly, another study identified married status as a positive prognostic factor in patients with squamous cell carcinoma of the oral cavity, oropharynx, hypopharynx and larynx. ${ }^{11}$ Several studies have also shown that the risk of mortality in the general population is lower for married patients. ${ }^{17-19}$ The mechanism for this survival advantage is unknown. It is possible that married patients have better social support and more financial resources than single patients, which can lead to improved compliance with treatment.

- Black race is a negative prognostic factor in a variety of head and neck cancers

- The impact of race on survival in localised papillary thyroid microcarcinoma patients was examined using a large population-based cancer database

- Black race is a negative prognostic factor in thyroid cancer, which cannot be explained by advanced disease stage

- The extent of surgery (total thyroidectomy vs lobectomy) did not affect survival in Black patients with papillary thyroid microcarcinoma

The main strength of our study lies in its large sample size and diverse patient characteristics. Utilising the Surveillance, Epidemiology, and End Results database allows us to analyse a large and diverse population, with outstanding quality control. The catchment areas used in the Surveillance, Epidemiology, and End Results database were selected for their ability to maintain a high-quality cancer reporting system and for demographic characteristics that are representative of the US population as a whole. This study has several limitations. It is limited by the retrospective nature of the analysis. The Surveillance, Epidemiology, and End Results database does not provide information on tumour size for cases diagnosed prior to 1988.
Consequently, these cases were excluded from the analysis. The database also does not include information on disease recurrence or treatments received beyond four months after diagnosis. ${ }^{20}$ Therefore, the impact of recurrence and subsequent treatment received cannot be examined.

In conclusion, our study shows that race influences survival outcomes for patients with papillary thyroid microcarcinoma. Black race is a negative prognostic factor, which cannot be explained by advanced disease stage. This finding is significant given the overall excellent prognosis expected of patients with papillary thyroid microcarcinoma. Further research on the mechanisms by which race affects survival is needed to reveal potential areas of opportunity for public health interventions aimed at reducing health disparities in cancer care.

\section{References}

1 Kilfoy BA, Zheng T, Holford TR, Han X, Ward MH, Sjodin A et al. International patterns and trends in thyroid cancer incidence, 1973-2002. Cancer Causes Control 2009;20: 525-31

2 Davies L, Welch HG. Increasing incidence of thyroid cancer in the United States, 1973-2002. JAMA 2006;295:2164-7

3 Davies L, Welch HG. Current thyroid cancer trends in the United States. JAMA Otolaryngol Head Neck Surg 2014;140: 317-22

4 Leenhardt L, Grosclaude P, Chérié-Challine L; Thyroid Cancer Committee. Increased incidence of thyroid carcinoma in France: a true epidemic or thyroid nodule management effects? Report from the French Thyroid Cancer Committee. Thyroid 2004;14: 1056-60

5 Dean DS, Hay ID. Prognostic indicators in differentiated thyroid carcinoma. Cancer Control 2000;7:229-39

6 Shaha AR. Implications of prognostic factors and risk groups in the management of differentiated thyroid cancer. Laryngoscope 2004;114:393-402

7 Jonklaas J, Nogueras-Gonzalez G, Munsell M, Litofsky D, Ain KB, Bigos ST et al.; National Thyroid Cancer Treatment Cooperative Study Group. The impact of age and gender on papillary thyroid cancer survival. J Clin Endocrinol Metab 2012;97: E878-87

8 Chen AY, Halpern M. Factors predictive of survival in advanced laryngeal cancer. Arch Otolaryngol Head Neck Surg 2007;133: $1270-6$

9 Shavers VL, Harlan LC, Winn D, Davis WW. Racial/ethnic patterns of care for cancers of the oral cavity, pharynx, larynx, sinuses, and salivary glands. Cancer Metastasis Rev 2003;22: 25-38

10 Olarte LS, Megwalu UC. The impact of demographic and socioeconomic factors on major salivary gland cancer survival. Otolaryngol Head Neck Surg 2014;150:991-8

11 de Graeff A, de Leeuw JR, Ros WJ, Hordijk GJ, Blijham GH, Winnubst JA. Sociodemographic factors and quality of life as prognostic indicators in head and neck cancer. Eur J Cancer 2001;37:332-9

12 Harari A, Li N, Yeh MW. Racial and socioeconomic disparities in presentation and outcomes of well-differentiated thyroid cancer. J Clin Endocrinol Metab 2014;99:133-41

$13 \mathrm{Yu}$ XM, Wan Y, Sippel RS, Chen H. Should all papillary thyroid microcarcinomas be aggressively treated? An analysis of 18,445 cases. Ann Surg 2011;254:653-60

14 Surveillance, Epidemiology, and End Results (SEER) Program (www.seer.cancer.gov) SEER*Stat Database: Incidence SEER 18 Regs Research Data + Hurricane Katrina Impacted Louisiana Cases, Nov 2011 Sub (1973-2009 varying) Linked To County Attributes - Total U.S., 1969-2010 Counties, National Cancer Institute, DCCPS, Surveillance Research Program, Surveillance Systems Branch, released April 2012, based on the November 2011 submission 
15 Moo-Young TA, Panergo J, Wang CE, Patel S, Duh HY, Winchester DJ et al. Variations in clinicopathologic characteristics of thyroid cancer among racial ethnic groups: analysis of a large public city hospital and the SEER database. Am J Surg 2013; 206:632-40

16 Noureldine SI, Abbas A, Tufano RP, Srivastav S, Slakey DP, Friedlander $\mathrm{P}$ et al. The impact of surgical volume on racial disparity in thyroid and parathyroid surgery. Ann Surg Oncol 2014; 21:2733-9

17 Murray JE. Marital protection and marital selection: evidence from a historical-prospective sample of American men. Demography 2000;37:511-21

18 Manzoli L, Villari P, Pirone GM, Boccia A. Marital status and mortality in the elderly: a systematic review and meta-analysis. Soc Sci Med 2007;64:77-94

19 Rendall MS, Weden MM, Favreault MM, Waldron H. The protective effect of marriage for survival: a review and update. Demography 2011;48:481-506
20 Cooper GS, Virnig B, Klabunde CN, Schussler N, Freeman J, Warren JL. Use of SEER-Medicare data for measuring cancer surgery. Med Care 2002;40:IV-43-48

Address for correspondence:

Dr Uchechukwu C Megwalu,

Department of Otolaryngology - Head and Neck Surgery,

Stanford University School of Medicine,

801 Welch Road,

Stanford, CA 94305, USA

Fax: +1 6507258502

E-mail:megwaluu@yahoo.com

Dr U C Megwalu takes responsibility for the integrity of the content of the paper

Competing interests: None declared 